\title{
Biodegradable in situ Gel for Subcutaneous Administration of Simvastatin for Osteoporosis
}

\author{
MONIKA N. SHEKHAWAT, ZUBIYA SURTII ${ }^{1}$ NAAZNEEN SURTI ${ }^{1 *}$ \\ Parul Institute of Pharmacy and Research, Waghodia, Vadodara-391 110, ${ }^{1}$ Babaria Institute of Pharmacy, BITS \\ Edu Campus, Vernama, Vadodara-391 240, India
}

Shekhawat, et al.: Biodegradable in situ Gel for Osteoporosis

\begin{abstract}
Many observational studies and animal research has revealed that statins appear to enhance osteoblastic activity and diminish osteoclast activity, hence increasing bone density. This study was aimed to prepare and characterize biodegradable in situ gel for subcutaneous administration of simvastatin for the treatment of osteoporosis. Biodegradable in situ gel was prepared using chitosan as biodegradable polymer and $\beta$-glycerol phosphate disodium salt hydrate as a buffering agent to achieve in situ gelation at physiological pH and temperature. In situ gel was characterized in terms of $\mathrm{pH}$, gelling capacity, drug content, sedimentation volume, resuspendability, syringeability, in vitro drug release, particle size and zeta potential. The optimized formulation, containing $1.25 \% \mathrm{w} / \mathrm{v}$ chitosan and $1 \% \beta$-glycerol phosphate disodium salt hydrate, had good gelling capacity at physiological temperature and $\mathrm{pH}$. It had a $\mathrm{pH}$ of $5.85 \pm 0.5$, drug content of $97.1 \pm 1.2 \%$ and sedimentation volume of $\mathbf{0 . 4 2} \pm \mathbf{0 . 0 4}$. In vitro drug release was found to be $98.7 \pm 1.5 \%$ in 7 days. Particle size, zeta potential and viscosity were found to be $7.07 \pm 0.3 \mu \mathrm{m}, 12.5 \pm 1.5 \mathrm{mV}$ and $22.66 \pm 1.8 \mathrm{cp}$, respectively. Hence, the developed biodegradable in situ gel administered subcutaneously can prove to be an effective alternative drug delivery system for the treatment of osteoporosis.
\end{abstract}

Key words: Simvastatin, in situ gel, osteoporosis, subcutaneous, biodegradable

Osteoporosis is a systemic skeletal disease characterized by low bone mass and micro architectural deterioration of bone tissue, which leads to bone fragility and susceptibility to fracture ${ }^{[1]}$. It is also called a "silent disease" as it develops without any noticeable symptoms until a fracture occurs ${ }^{[2]}$. Statin drugs are clinically used in condition of hyperlipidaemia to lower the cholesterol levels and therefore reducing the risk of heart attack. However, many studies revealed that statins appear to enhance osteoblastic activity by both increasing expression of bone morphogenetic protein-2, a stimulator of osteoblast differentiation, and diminishing osteoclast activity by preventing prenylation and activation of key intracellular proteins $^{[1]}$. All the available data from the literature, which include, evidence from experimental studies as well as from the vast majority of observational studies and the results of a single meta-analysis, suggest that there is a positive effect of statins on bone mass density ${ }^{[3]}$.

It has been observed that the response to increase bone formation in rats was greater when the statins were administered transdermally as compared to oral

\footnotetext{
This is an open access article distributed under the terms of the Creative Commons Attribution-NonCommercial-ShareAlike 3.0 License, which allows others to remix, tweak, and build upon the work non-commercially, as long as the author is credited and the new creations are licensed under the identical terms
}

Accepted 27 February 2018

Revised 12 August 2017

Received 14 December 2016

Indian J Pharm Sci 2018;80(2):395-399 
administration of the same. This further depends on potency and lipophilicity of each statin. Simvastatin has a positive effect on bone mass density. Moreover it also has enough lipophilicity to enter the cells easily ${ }^{[4]}$. Biodegradable injectable in situ gel forming drug delivery systems represent an attractive alternative to microspheres and implants as parenteral depot systems. It consists of biodegradable polymers dissolved in a biocompatible carrier. When the liquid polymer system is placed in the body using standard needles and syringes, it undergoes solidification upon contact with aqueous body fluids to form solid implant. If a drug is incorporated into the polymer solution, it becomes entrapped within polymer matrix as it solidifies. Drug release occurs over time as polymer biodegrades. Biodegradable polymers used in these systems are polyanhydrides, polyhydroxy acids, polyesteramides, polyorthoesters and others ${ }^{[5]}$. Chitosan (CS) is an aminopolysaccharide obtained by alkaline deacetylation of chitin, a natural component of shrimp or crab shells. It is biocompatible and biodegradable $\mathrm{pH}$-dependent cationic polymer. Polyol salts bearing a single anionic head, such as glycerol-, sorbitol-, fructose- or glucosephosphate salts (polyol- or sugar-phosphates) are ideal agents for transforming purely $\mathrm{pH}$-dependent $\mathrm{CS}$ solutions into temperature-controlled $\mathrm{pH}$-dependent $\mathrm{CS}$ solutions. The combination of $\mathrm{CS}$, a cationic polysaccharide, and polyol-phosphate salts is beneficial from several synergistic forces favourable to gel formation including hydrogen bonding, electrostatic interactions and hydrophobic interactions. This set of phosphate salts gives a unique behaviour by allowing the CS solutions to remain liquid at the physiological $\mathrm{pH}$ and to turn into gel if heated at body temperature ${ }^{[5-7]}$.

Hence, an attempt was made to prepare and characterize biodegradable in situ gel for subcutaneous administration of a simvastatin, which could be useful in the treatment of osteoporosis. CS (degree of deacetylation of approximately $91 \%$ ) was procured from Chemdyes Corporation, Vadodara. $\beta$-Glycerol phosphate disodium salt hydrate ( $\beta$-GP) was purchased from Sigma Aldrich (USA). Simvastatin was purchased from Centurion Laboratories (Vadodara). All the other chemicals and reagents used were of analytical grade.

CS was dissolved in $0.1 \mathrm{~N} \mathrm{HCl}$ and the mixture was stirred vigorously till a clear solution was obtained. CS solution was chilled to a temperature of $4-5^{\circ} . \beta$-GP was dissolved in distilled water. It was then cooled to $4-5^{\circ}$ and was subsequently added to chilled CS solution in a controlled manner. The resulting mixture was stirred for
$5 \mathrm{~min}$. The calculated amount of drug was weighed and dispersed, and then sonicated for $10 \mathrm{~s}$. Propyl paraben $(0.02 \%)$ was used as a preservative. This in situ gel was sterilised by autoclaving at $121^{\circ}$ for $15 \mathrm{~min}$. The concentration of CS and $\beta$-GP were optimised by using manual experimental design as shown in Table 1. $\mathrm{pH}$ of each formulation was determined by using digital $\mathrm{pH}$ meter. It has been reported that the discomfort at injection site would be minimum, if the $\mathrm{pH}$ is close to the physiological $\mathrm{pH}$.

Gelling systems of various concentrations of CS and $\beta$-GP were prepared and evaluated for gelling capacity in order to identify the compositions suitable for use as an in situ gelling system. The gelling capacity was determined by placing $100 \mu \mathrm{l}$ of in situ gel in a vial containing $2 \mathrm{ml}$ of phosphate buffer at $\mathrm{pH} 7.4$ and equilibrated at $37 \pm 1^{\circ}$ and visually assessing the gel formation and noting the time for gelation. +: Gels after few minutes, dissolves rapidly, ++ : immediate gelation and remains for few hours, +++ : immediate gelation and remains for extended periods.

To determine drug content, an amount of in situ gel containing a specified amount of drug was taken and dissolved in $10 \mathrm{ml}$ of methanol. Appropriate dilutions were made using phosphate buffer $\mathrm{pH}$ 7.4. The absorbance was measured spectrophotometrically at $238 \mathrm{~nm}$. About $10 \mathrm{ml}$ of the suspension was transferred to a stoppered measuring cylinder and was stored at room temperature for $72 \mathrm{~h}$. The volume of sediment formed was noted. The sedimentation volume was calculated as the ratio of ultimate height $(\mathrm{Hu})$ of the sediment to the final height $(\mathrm{Ho})$ of the suspension $^{[8]}$. Sedimentation volume $=\mathrm{Hu} / \mathrm{Ho}$. After sedimentation was complete, the measuring cylinder was manually shaken. Based on the effort and number of shakings needed to convert the sedimented system to a homogenous suspension, the prepared product was rated as, resuspendable, resuspendable with difficulty or not resuspendable ${ }^{[8]}$.

Particle size was determined using optical microscopic method, before and after sonication of the formulation. Zetasizer (Nano-ZS, Malvern Instruments, UK) was used to determine the zeta potential. Viscosity of all the batches was determined using a Brookfield viscometer. The viscosity was measured at $50 \mathrm{rpm}$ and room temperature using spindle number 3 .

In vitro release of the drug from in situ gel was measured using the membrane less diffusion method. From each formulation, $2 \mathrm{ml}$ solution containing the 
drug was transferred into a beaker containing $150 \mathrm{ml}$ of $\mathrm{pH} 7.4$ phosphate buffer maintained at $37 \pm 1^{\circ}$. As the solution came into contact with the buffer, it transformed in to a gel. About $5 \mathrm{ml}$ of samples were collected at predetermined time intervals, replacing fresh $5 \mathrm{ml}$ buffer into the dissolution medium. Agitation was maintained in the beaker with the help of a magnetic stirrer. The drug concentration was determined spectrophotometrically at $238 \mathrm{~nm}^{[9]}$.

Syringeability was determined employing two methods. Qualitative measurement of syringeability in which the formulation was tested by passing through a 24-gauge needle to test the ease of administration. Second method was syringeability measurement using the Brookfield's Texture analyser, QTS.

Sterility testing was performed using the fluid thioglycollate and soybean-casein digest medium with direct inoculation method, as per the Indian Pharmacopoeia. About $2 \mathrm{ml}$ of the sample was taken from sterilized sample with the help of sterile pipette or syringe and was transferred aseptically to fluid thioglycollate medium and soybean-casein digest medium separately. These samples were incubated for $14 \mathrm{~d}$ at $30-35^{\circ}$ in case of fluid thioglycollate medium and $20-25^{\circ}$ in case of soybean-casein digest medium. The test tubes were checked for growth everyday till $14 \mathrm{~d}$. $\mathrm{pH}$ of each formulation was determined by using digital $\mathrm{pH}$ meter. As the concentration of $\beta$-GP increased it neutralized the CS solution and increased the $\mathrm{pH}$ to bring it near physiological $\mathrm{pH} . \mathrm{pH}$ values of all the formulation batches were shown in Table 2.

TABLE 1: COMPOSITION OF EXPERIMENTAL BATCHES

\begin{tabular}{lcc}
\hline Batches & CS (\% w/v) & B-GP $(\%$ w/v) \\
\hline A1 & & 1.0 \\
A2 & 0.5 & 2.0 \\
A3 & & 3.0 \\
A4 & & 4.0 \\
B1 & 1.0 \\
B2 & 0.75 & 2.0 \\
B3 & & 3.0 \\
B4 & & 4.0 \\
C1 & & 1.0 \\
C2 & 1.0 & 2.0 \\
C3 & & 3.0 \\
C4 & & 4.0 \\
D1 & & 1.0 \\
D2 & 1.25 & 2.0 \\
D3 & & 3.0 \\
D4 & & 4.0 \\
\hline B-GP: B-gycel & &
\end{tabular}

B-GP: B-glycerol phosphate disodium salt hydrate; CS: chitosan
The gelling capacity was determined by placing $100 \mu \mathrm{l}$ of in situ gel in a test tube containing $2 \mathrm{ml}$ of phosphate buffer at $\mathrm{pH} 7.4$ and equilibrated at $37 \pm 1^{\circ}$ and visually assessing the gel formation and noting the time for gelation. The increase in the gelling capacity of the in situ gel was directly related to the concentration of the CS. The increase in amount of CS lead to an increase in the gelling capacity of the in situ gel. Gelling capacity of all the formulation batches are shown in Table 2. In situ gel is shown in fig. 1a. Fig. 1b depicted the formation of gel after placing it in $\mathrm{pH} 7.4$ buffer. To determine drug content, the amount of in situ gel containing specific amount of drug was taken and dissolved in $10 \mathrm{ml}$ of methanol. Appropriate dilutions were made using $\mathrm{pH} 7.4$ buffer. This solution was analysed using $\mathrm{UV} /$ Vis spectrophotometer at $238 \mathrm{~nm}$. The results were presented in Table 2.

About $10 \mathrm{ml}$ of the suspension was transferred to a stoppered measuring cylinder and was stored at room temperature for $72 \mathrm{~h}$. The volume of sediment formed was noted. The sedimentation volume $\mathrm{Hu} / \mathrm{Ho}$ was calculated. The sedimentation volume increased as the concentration of CS increased in the formulation as shown in Table 2. As the concentration of CS increased the viscosity also increased, which led to a slower sedimentation of the particles. Change in the concentration of $\beta$-GP was not found to affect the sedimentation volume. The resuspendability potential of the sediment was tested and the formulation batches were found to be resuspendable even after a period of $1 \mathrm{w}$.

The particle size of all the batches, before and after sonication, was determined. Correction factor = (number of divisions of eyepiece micrometre/number of divisions of stage micrometre $) \times 10$. Correction factor $=$ $(15 / 11) \times 10=13.63$. As recorded in Table 2 , all batches, after sonication, had a particle size below $10 \mu \mathrm{m}$, which was not expected to block the needle and hence expected to have good syringeability. Zeta potential of the optimized batch was found to be $12.5 \pm 1.5 \mathrm{mV}$, which indicated stability of the formulation. Viscosity was measured at $50 \mathrm{rpm}$ and room temperature using spindle number 3 . The viscosity of all the batches was shown in Table 2 . The viscosity of the in situ gel increased as there is increase in the concentration of CS. The viscosity of the formulation directly influenced the syringeability.

In vitro release of drug from in situ gel was evaluated using the membrane less diffusion method as already described. The drug concentration was deduced 
from the calibration curve of pure drug in different dissolution media. The in vitro drug release of all the batches was presented in Table 3. Batches A1 to A4 released the drug within $24 \mathrm{~h}, \mathrm{~B} 1$ to $\mathrm{B} 4$ showed drug release up to $48 \mathrm{~h}$, batches $\mathrm{C} 1$ to $\mathrm{C} 4$ could extend the drug release up to $5 \mathrm{~d}$ and D1 to D4 showed extended drug release for $7 \mathrm{~d}$. As the CS concentration was increased, there was decrease in the drug release rate. The reason for this response could be the formation of swollen gel like structure that could substantially reduce the penetration of dissolution medium into the gel. Increase in the concentration of CS led to the formation of more dense gel from which the drug release was retarded. The optimised formulation followed Higuchi square root kinetic model, which indicated that drug release followed diffusion mechanism. The peak load and mean load for syringeability of the optimized batch was found to be $974.12 \mathrm{~g}$ and $354.23 \mathrm{~g}$, respectively as shown in fig. 2.

Sterility testing was performed using the fluid thioglycolate and soybean-casein digest medium, respectively. No microbial growth (turbidity) was observed in both the media. As there was no microbial growth in the sterilized media containing the formulation stored at the above-mentioned conditions, it was interpreted that the formulations were sterile.

Results of gelling capacity (Table 2) and drug release (Table 3) revealed that batches containing $1.25 \% \mathrm{w} / \mathrm{v}$ CS (D1 to D4) had good gelling capacity (showed immediate gelation and remained for extended periods) and could sustain the drug release for up to $7 \mathrm{~d}$. Increasing the concentration of $\beta$-GP from 1.0 to $4.0 \% \mathrm{w} / \mathrm{v}$ did not show any significant difference in results, hence D1 $(1.25 \% \mathrm{w} / \mathrm{v}$ CS and $1.0 \% \mathrm{w} / \mathrm{v} \beta-\mathrm{GP})$ was considered as optimized formulation.

In the present study, biodegradable in situ gel containing simvastatin, which was intended to be administered subcutaneously, was prepared using CS as the polymer and $\beta$-GP as the buffering agent. The concentration of CS and $\beta$-GP were optimized successfully using manual experimental design. $1.25 \% \mathrm{CS}$ and $1 \% \beta$-GP concentrations were selected as optimum formulation parameters. The $\mathrm{pH}$, drug content, sedimentation volume, in vitro drug release, particle size, zeta potential and viscosity of the optimised formulation

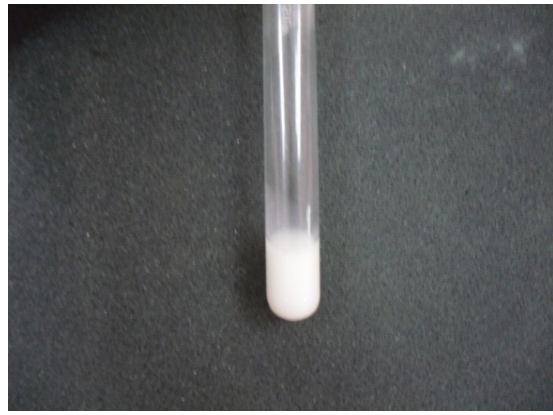

b.

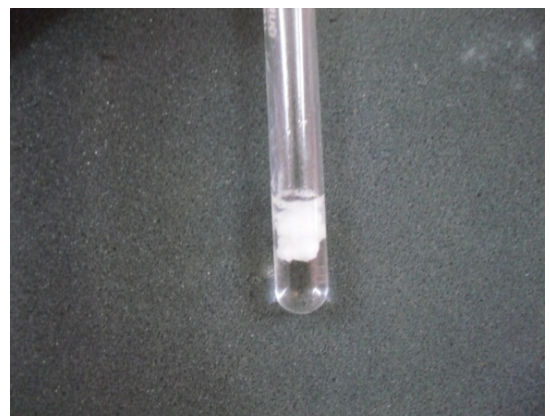

Fig. 1: In situ gel at room conditions (a) and in situ gel formation in pH 7.4 buffer (b)

TABLE 2: EVALUATION OF DIFFERENT BATCHES OF IN SITU GEL

\begin{tabular}{ccccccc}
\hline Batch & pH & Gelling capacity & Sedimentation volume & Viscosity $(\mathrm{Cps})$ & Particle size $(\mu \mathrm{m})$ & Drug content $(\%)$ \\
\hline A1 & 4.34 & + & 0.14 & $9.33 \pm 1.5$ & $6.73 \pm 0.15$ & $95.4 \pm 1.04$ \\
A2 & 5.3 & + & 0.15 & $8.66 \pm 1.5$ & $6.98 \pm 0.06$ & $93.74 \pm 0.29$ \\
A3 & 5.46 & + & 0.13 & $8.5 \pm 1.0$ & $6.99 \pm 0.11$ & $97.15 \pm 0.50$ \\
A4 & 6.16 & - & 0.14 & $8.9 \pm 1.0$ & $7.02 \pm 0.04$ & $93.31 \pm 0.60$ \\
B1 & 4.18 & ++ & 0.22 & $11.33 \pm 0.5$ & $6.98 \pm 0.04$ & $97.81 \pm 0.80$ \\
B2 & 5.95 & ++ & 0.23 & $10.66 \pm 1.0$ & $7.03 \pm 0.11$ & $96.77 \pm 0.36$ \\
B3 & 6.27 & ++ & 0.22 & $11.6 \pm 0.5$ & $6.97 \pm 0.11$ & $94.40 \pm 0.44$ \\
B4 & 6.73 & ++ & 0.22 & $10.9 \pm 0.5$ & $7.016 \pm 0.11$ & $86.27 \pm 1.02$ \\
C1 & 5.34 & ++ & 0.30 & $16 \pm 2.0$ & $6.75 \pm 0.04$ & $97.95 \pm 0.53$ \\
C2 & 5.75 & ++ & 0.32 & $15.33 \pm 1.0$ & $6.94 \pm 0.06$ & $91.23 \pm 0.74$ \\
C3 & 6.46 & ++ & 0.30 & $16.5 \pm 1.5$ & $7.02 \pm 0.04$ & $88.89 \pm 0.65$ \\
C4 & 6.81 & ++ & 0.31 & $16 \pm 0.0$ & $6.86 \pm 0.07$ & $92.62 \pm 0.67$ \\
D1 & 5.85 & +++ & 0.42 & $22.66 \pm 1.5$ & $7.07 \pm 0.04$ & $97.1 \pm 1.2$ \\
D2 & 6.17 & +++ & 0.42 & $21.33 \pm 2.5$ & $6.95 \pm 0.02$ & $91.1 \pm 0.61$ \\
D3 & 6.45 & +++ & 0.40 & $21 \pm 1.5$ & $7.04 \pm 0.02$ & $96.26 \pm 0.65$ \\
D4 & 6.71 & +++ & 0.41 & $22.3 \pm 1.0$ & $6.97 \pm 0.06$ & $95.37 \pm 0.44$ \\
\hline
\end{tabular}


www.ijpsonline.com

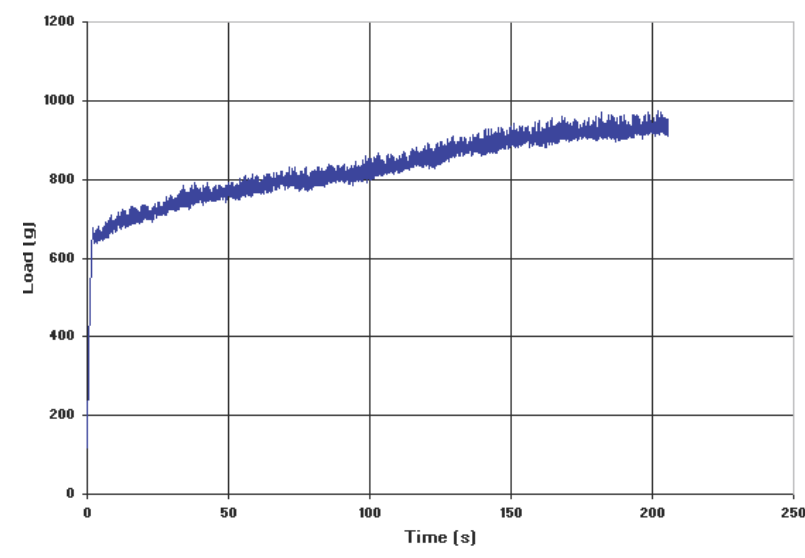

Fig. 2: Load v/s time graph for syringeability

TABLE 3: CUMULATIVE DRUG RELEASED FROM DIFFERENT BATCHES OF IN SITU GEL

\begin{tabular}{lc}
\hline Batch no. & Cumulative drug release (\%) \\
\hline A1 & $97.26 \pm 0.56$ (in $24 \mathrm{~h})$ \\
A2 & $98.32 \pm 0.95$ (in $24 \mathrm{~h})$ \\
A3 & $97.89 \pm 0.72$ (in $24 \mathrm{~h})$ \\
A4 & $98.18 \pm 0.71$ (in $24 \mathrm{~h})$ \\
B1 & $99.09 \pm 0.87$ (in $48 \mathrm{~h})$ \\
B2 & $98.03 \pm 0.56$ (in $48 \mathrm{~h})$ \\
B3 & $98.99 \pm 0.49$ (in $48 \mathrm{~h})$ \\
B4 & $98.95 \pm 0.59$ (in $48 \mathrm{~h})$ \\
C1 & $98.04 \pm 0.92$ (in $5 \mathrm{~d})$ \\
C2 & $98.45 \pm 0.31$ (in $5 \mathrm{~d})$ \\
C3 & $98.00 \pm 0.47$ (in $5 \mathrm{~d})$ \\
C4 & $98.40 \pm 0.97$ (in $5 \mathrm{~d})$ \\
D1 & $98.76 \pm 0.59$ (in $7 \mathrm{~d})$ \\
D2 & $97.35 \pm 0.28$ (in $7 \mathrm{~d})$ \\
D3 & $96.54 \pm 0.34$ (in $7 \mathrm{~d}$ ) \\
D4 & $97.14 \pm 0.27$ (in $7 \mathrm{~d}$ ) \\
\hline
\end{tabular}

were found to be $5.85 \pm 0.5,97.1 \pm 1.2,0.42 \pm 0.04$, $98.7 \pm 1.5 \%$ cumulative drug release in $7 \mathrm{~d}, 7.07 \pm 0.3$ $\mu \mathrm{m}, 12.5 \pm 1.5 \mathrm{mV}$ and $22.66 \pm 1.8 \mathrm{cp}$, respectively. The optimised in situ gel showed immediate gelation and retained the gel form up to $7 \mathrm{~d}$. Hence, the developed biodegradable in situ gel of simvastatin can be effectively used for subcutaneous administration in the treatment of osteoporosis.

\section{Conflict of interest:}

The authors declare no conflicts of interest.

\section{Financial support and sponsorship:}

Nil.

\section{REFERENCES}

1. Cruz AC, Gruber BL. Statins and osteoporosis: Can these lipid-lowering drugs also bolster bones? Cleve Clin J Med 2002;69(4):277-88.

2. http://www.endocrineweb.com/conditions/osteoporosis/ osteoporosis-symptoms.

3. Tsartsalis AN, Dokos C, Kaiafa GD, Tsartsalis DN, Kattamis A, Hatzitolios AI, et al. Statins, bone formation and osteoporosis: hope or hype? Hormones 2012;11(2):126-39.

4. Lupatelli G, Scarponi AM, Vaudo G, Siepi D, Roscini AR, Gemelli F, et al. Simvastatin increases bone mineral density in hypercholesterolaemia postmenopausal women. Metabolism 2004;53(6):744-48.

5. Chenite A, Chaput C, Wang D, Combes C, Buschmann $\mathrm{MD}$, Hoemann CD, et al. Novel injectable neutral solutions of chitosan form biodegradable gels in situ. Biomaterials 2000;21:2155-61.

6. Sharma G, Italia JL, Sonaje K, Tikoo K, Ravikumar MNV. Biodegradable in situ gelling system for subcutaneous administration of ellagic acid and ellagic acid loaded nanoparticles: Evaluation of their antioxidant potential against cyclosporine-induced nephrotoxicity in rats. J Control Release 2007;118:27-37.

7. Wu H, Liu Z, Peng J, Li L, Li N, Li J, et al. Design and evaluation of baicalin-containing in situ ph-triggered gelling system for sustained ophthalmic drug delivery. Int J Pharm 2011;410:31-40.

8. Sabri LA, Rasool AA and Shehab MA. Formulation of Rifampicin suspension. Iraqi J Pharm Sci 2006;15:1-7.

9. Korsmeyer RW, Gumy R, Doelker E, Buri P. Peppas NA. Mechanisms of solute release from porous hydrophilic polymers. Int J Pharm 1983;15:25-35. 